\title{
The triangular fibrocartilage complex on high-resolution 3 T MRI in healthy adolescents: the thin line between asymptomatic findings and pathology
}

\author{
Anne-Sophie van der Post ${ }^{1,2,3}$ (D) $\cdot$ Sjoerd Jens $^{4} \cdot$ Frank F. Smithuis ${ }^{1} \cdot$ Miryam C. Obdeijn $^{2,5} \cdot$ Roelof-Jan Oostra $^{6}$. \\ Mario Maas ${ }^{1,2,3}$
}

Received: 4 January 2021 / Revised: 31 March 2021 / Accepted: 31 March 2021 / Published online: 17 April 2021

(C) The Author(s) 2021

\begin{abstract}
Objective The objective of the study is to provide a reference for morphology, homogeneity, and signal intensity of triangular fibrocartilage complex (TFCC) and TFCC-related MRI features in adolescents.

Materials and methods Prospectively collected data on asymptomatic participants aged 12-18 years, between June 2015 and November 2017, were retrospectively analyzed. A radiograph was performed in all participants to determine skeletal age and ulnar variance. A 3-T MRI followed to assess TFCC components and TFCC-related features. A standardized scoring form, based on MRI definitions used in literature on adults, was used for individual assessment of all participants by four observers. Results per item were expressed as frequencies (percentages) of observations by all observers for all participants combined $(n=92)$. Interobserver agreement was determined by the unweighted Fleiss' kappa with 95\% confidence intervals (95\% CI).

Results The cohort consisted of 23 asymptomatic adolescents (12 girls and 11 boys). Median age was 13.5 years (range 12.017.0). Median ulnar variance was $-0.7 \mathrm{~mm}$ (range-2.7-1.4). Median triangular fibrocartilage (TFC) thickness was $1.4 \mathrm{~mm}$ (range 0.1-2.9). Diffuse increased TFC signal intensity not reaching the articular surface was observed in 30 (33\%) observations and a vertical linear increased signal intensity with TFC discontinuation in $19(20 \%)$ observations. Discontinuation between the volar radioulnar ligament and the TFC in the sagittal plane was seen in $23(25 \%)$ observations. The extensor carpi ulnaris was completely dislocated in $10(11 \%)$ observations, more frequent in supinated wrists $(p=0.031)$. Inter-observer agreement ranged from poor to fair for scoring items on the individual TFCC components.

Conclusion MRI findings, whether normal variation or asymptomatic abnormality, can be observed in TFCC and TFCC-related features of asymptomatic adolescents. The rather low inter-observer agreement underscores the challenges in interpreting these

Anne-Sophie van der Post

a.vanderpost@amsterdamumc.nl

small structures on MRI. This should be taken into consideration when interpreting clinical MRIs and deciding upon arthroscopy.
\end{abstract}

1 Department of Radiology and Nuclear Medicine, Amsterdam Movement Sciences, Amsterdam UMC, University of Amsterdam, Meibergdreef 9, Amsterdam, The Netherlands

2 Academic Center for Evidence-based Sports medicine (ACES), Amsterdam, The Netherlands

3 Amsterdam Collaboration for Health and Safety in Sports (ACHSS), International Olympic Committee (IOC) Research Center AMC/ VUmc, Amsterdam, The Netherlands

4 Department of Radiology and Nuclear Medicine, Rijnstate Hospital, Arnhem, The Netherlands

5 Department of Plastic, Reconstructive and Hand Surgery, Amsterdam Movement Sciences, Amsterdam UMC, University of Amsterdam, Meibergdreef 9, Amsterdam, The Netherlands

6 Department of Medical Biology, Section Clinical Anatomy and Embryology, Amsterdam UMC, University of Amsterdam, Meibergdreef 9, Amsterdam, The Netherlands
Keywords Triangular fibrocartilage $\cdot$ Adolescent $\cdot$ Magnetic resonance imaging · Wrist injuries

\section{Introduction}

The triangular fibrocartilage complex (TFCC) is a complex of fibro-cartilaginous and ligamentous structures, located between the distal ulna and carpal bones, facilitating distal radioulnar joint (DRUJ) stabilization, carpal stabilization, and axial load transmission [1]. TFCC injury can be sustained by trauma typically due to a fall on the outstretched hand, which is common in adults and children, yet it can also result from degeneration due to repetitive forces on the wrist in young athletes and adults $[2,3]$. It is generally considered a 
common cause of ulnar-sided wrist pain [3, 4]. However, ulnar-sided wrist pain comprises a challenging diagnostic process due to the wide differential diagnosis for this small anatomic region [5].

After physical examination, the first diagnostic step in ulnar-sided wrist pain is a standardized radiograph. This can show TFCC injury associated abnormalities such as ulnar styloid process fractures, positive ulnar variance, or degenerative changes $[4,6]$. Magnetic resonance imaging (MRI) can be performed when a clinical suspicion of TFCC injury remains. Arthroscopy, the diagnostic reference standard, is performed only when prompted by physical examination combined with MRI abnormalities [7].

However, clinicians should be aware of the high prevalence of TFCC "abnormalities" on MRI in the asymptomatic population ranging from $19 \%$ in populations under 30 years to $64 \%$ in populations over 70 years $[8,9]$. Additionally, MRI has remained controversial due to significant variations in diagnostic accuracy [10]. This can be explained by differences in observer experience and MRI quality as well as by the wide variation in definitions and criteria for diagnosing TFCC injury $[8,11-13]$.

Once thought rarely to occur in pediatric patients, arthroscopy recently showed TFCC tears in $48.5-80.5 \%$ of pediatric patients with persistent wrist pain [14-16]. Despite the absence of knowledge regarding TFCC features and its normal variation on MRI in children and adolescents, an increasing number of articles on surgical techniques and outcomes for TFCC injury have been published since [17-21]. We are not aware of any studies on TFCC features on MRI in adolescents and hypothesize that TFCC abnormalities are also prevalent in the asymptomatic pediatric population. Therefore, we assessed MRIs of healthy asymptomatic adolescents in order to obtain a reference for morphology, homogeneity, and signal intensity of the individual TFCC components and TFCCrelated features. This will assist in addressing MRI findings of the TFCC in patients with ulnar-sided wrist pain as either pathological or non-pathological and accordingly improve TFCC injury diagnosis.

\section{Materials and methods}

This observational cross-sectional study is a retrospective analysis of prospectively collected study data from adolescents involved in the Physeal MRI study that selected gymnasts with wrist pain, asymptomatic gymnasts, and healthy participants aged 12-18 years in Amsterdam University Medical Center, Location AMC, from June 2015 to November 2017 [22]. The current study only included the healthy participants. The study was performed in accordance with the Declaration of Helsinki and approved by the institution's Medical Review Ethics Committee (reference no.
2014 382). Written informed consent was given by each participant as well as the parent or legal guardian.

\section{Population}

Healthy girls and boys aged $12-18$ years without wrist pain over the last 6 months were included. Participants were not eligible if they had participated in gymnastics or performed wrist-loading sports over two times a week, had fused distal radial physes, were diagnosed with growth disturbance or musculoskeletal diseases, or had a history of wrist fracture, surgery, or infection.

\section{Imaging}

All participants underwent a radiograph followed by MRI of one wrist. Conventional posterior-anterior radiographs were obtained in $90^{\circ}$ shoulder abduction, $90^{\circ}$ elbow flexion, and neutral forearm position (focus-detector distance $1.30 \mathrm{~m}$ ). Skeletal age was determined using the validated BoneXpert software (v2.0.1.3; Visiana, Holte, Denmark). Ulnar variance was assessed on the radiographs by one specialized musculoskeletal radiologist (SJ) using the recommended perpendicular method [23]. Negative ulnar variance indicates a relatively shorter ulna. MRIs were obtained with a 3-T MRI scanner (Ingenia, Philips Healthcare, Best, The Netherlands) in a feet-first, supine position with the wrist placed neutral, alongside the body, using a dedicated wrist coil (eight channel, receive-only). The analyzed MRI sequences included turbo spin-echo (TSE) proton-density (PD) weighted sequences in three planes without fat saturation (1500-2000 ms repetition time (TR), $20 \mathrm{~ms}$ echo time (TE), $90^{\circ}$ flip angle, $2.5-1.5 \mathrm{~mm}$ slice thickness, $0.30 \times 0.30 \mathrm{~mm}$ spatial resolution, 4:064:39 min scan time), a TSE PD weighted coronal sequence with fat saturation by spectral attenuated inversion recovery (SPAIR) (2000 ms TR, $30 \mathrm{~ms} \mathrm{TE}, 90^{\circ}$ flip angle, $2.5 \mathrm{~mm}$ slice thickness, $0.30 \times 0.32 \mathrm{~mm}$ spatial resolution, $4: 12 \mathrm{~min}$ scan time), and a TSE T2 weighted axial sequence with fat saturation by SPAIR $\left(3001 \mathrm{~ms}\right.$ TR, $60 \mathrm{~ms}$ TE, $90^{\circ}$ flip angle, $2.5 \mathrm{~mm}$ slice thickness, $0.30 \times 0.36 \mathrm{~mm}$ spatial resolution, 4:58 min scan time).

\section{Standardized scoring form}

A standardized scoring form focusing on observation of morphology, homogeneity, and signal intensity and explicitly not on detecting pathology was constructed by a physician experienced in research on musculoskeletal imaging (AP). Due to the lack of a consensus reference standard regarding the individual TFCC components, the items were based on MRI definitions used by Zhan et al. on adults, i.e., triangular fibrocartilage (TFC), dorsal and volar radioulnar ligaments (RUL), meniscus homolog, extensor carpi ulnaris (ECU) 
tendon sheath, ulnocarpal ligaments (ulnolunate and ulnotriquetral), and ulnar collateral ligament [24]. Images from trial cases that were not included in the present study (i.e., symptomatic gymnasts) were provided as illustrative examples in the scoring form (see supplementary material).

First, scoring items were adjusted, added, and deleted based on clinical relevance and feasibility in several rounds of consensus meetings with a single-center expert group of three musculoskeletal radiologists (MM, FS en SJ with 26, 5 and 1 years of experience, respectively) and one hand surgeon (MO with 15 years of experience). Items on ulnocarpal ligaments and the ulnar collateral ligament were deleted due to lack of clinical relevance and controversy over their existence [25]. The recommended sequences and planes for assessment were determined in consensus and stated per item. The final score form, as provided by the supplementary material, was piloted in several trial cases for calibration prior to scoring.

Then, each member of the expert group individually assessed the blinded MRI scans in a randomized order on a diagnostic PC workstation with a high-resolution monitor using the IMPAX software version 6.6.1.4024 (AGFA HealthCare N.V., Mortsel, Belgium). In order to prevent response fatigue, observers were allowed to assess only three consecutive MRIs followed by a mandatory break. Each observer was blinded for other observers' scoring results and observers were aware that subjects were asymptomatic. Windowing, zooming, and scrolling through the images was allowed.

\section{Statistical analysis}

Patient characteristics and scoring form observations were entered into Castor Electronic Data Capture (Ciwit BV, Amsterdam, The Netherlands, 2018) and directly imported for analysis in RStudio (RStudio, Inc., Boston). Descriptive statistics and TFC thickness were illustrated as medians with ranges. TFC thickness measurements that were not possible due to disc disruption were excluded from this analysis. An intraclass correlation coefficient (ICC) for inter-observer agreement on TFC thickness was calculated using a twoway random-effects (agreement, single measures) model. For each MRI characteristic, categorical variables scored by all observers for all participants were combined and expressed in frequencies of observations with percentages $(n=92)$. Inter-observer agreement was determined by the unweighted Fleiss' kappa with $95 \%$ confidence intervals $(95 \%$ CI). All correlation coefficients were interpreted as poor $(\leq 0.20)$, fair (0.21-0.40), moderate $(0.41-0.60)$, substantial $(0.61-0.80)$, or excellent $(0.81-1.00)$ agreement [26]. Differences were calculated with a Mann-Whitney $U$ test. $P$ values $<0.05$ were considered statistically significant.

\section{Results}

\section{Patient characteristics}

The study cohort consisted of 23 healthy adolescents and their demographic information is depicted in Table 1. One boy was excluded due to insufficient image quality based on motion artifacts.

\section{TFCC components}

All results for MRI characteristics of TFCC components are shown in Table 2.

\section{Triangular fibrocartilage (TFC)}

Median TFC thickness was $1.4 \mathrm{~mm}$ (range 0.1-2.9). Agreement between observers for measuring TFC thickness was moderate $(\mathrm{ICC}=0.60,95 \%$ confidence interval $=0.40$ $0.77)$. In the coronal plane, TFC morphology was identified as a slightly radially tilted asymmetrical bowtie in $46(50 \%)$ observations, as a shorter, thicker, and more horizontal structure in $34(37 \%)$ and as a thinner and more stretched structure in only $12(13 \%)$ (Fig. 1). We found no statistical difference in wrist position $(p=0.26)$ or ulnar variance $(p=0.17)$ for wrists where a thinner and more stretched TFC configuration was observed. In the sagittal plane, TFC morphology was scored as a symmetrical biconcave disc in $48(52 \%)$ and as an asymmetrical disc being thicker at the dorsal side than at the volar side in $41(45 \%)$ observations. Inter-observer agreement for scoring coronal morphology was fair $(\mathrm{ICC}=0.33,95 \% \mathrm{CI}=$ $0.20-0.46)$ and for scoring sagittal morphology was poor $(\mathrm{ICC}=0.19,95 \% \mathrm{CI}=0.20-0.53)$.

TFC homogeneity in the coronal plane was scored as a homogeneous hypo-intense signal intensity in 43 (47\%) and as a diffuse increased signal intensity not reaching the articular surface in 30 (33\%) (Fig. 2). In 14 (15\%) observations regarding TFC homogeneity, a vertical linear increased signal intensity with discontinuation of the TFC was identified. Other

Table 1 Participant demographics

\begin{tabular}{ll}
\hline Items & Number (percentage) \\
\hline Participants & $23(100)$ \\
Sex (girls/boys) & $12(52) / 11(48)$ \\
Wrist (right/left) & $13(57) / 10(43)$ \\
& \\
Calendar age (years) & Median (range) \\
Skeletal age (years) & $13.5(12.0-17.0)$ \\
Ulnar variance (millimeters) & $13.4(11.3-17.6)$ \\
\hline
\end{tabular}


Table 2 MRI characteristics scored by all observers in frequencies of observations for all participants by all observers combined with percentages calculated from the total amount of observations $(n=92)$

\begin{tabular}{|c|c|c|c|}
\hline Scoring item & MRI characteristics & Frequency $(\%)$ & Kappa (95\% CI) \\
\hline \multicolumn{4}{|l|}{ Triangular fibrocartilage } \\
\hline \multirow[t]{3}{*}{ Coronal morphology } & Slightly radially tilted bowtie & $46(50)$ & $0.33(0.20-0.46)$ \\
\hline & Shorter, thicker and more horizontal & $34(37)$ & \\
\hline & Thinner and more stretched & $12(13)$ & \\
\hline \multirow[t]{4}{*}{ Coronal homogeneity } & Homogeneous hypo-intense & $43(47)$ & $0.13(0.16-0.24)$ \\
\hline & Diffuse increased SI & $30(33)$ & \\
\hline & Vertical linear increased SI & $14(15)$ & \\
\hline & Other & $5(5)$ & \\
\hline \multirow[t]{3}{*}{ Sagittal morphology } & Symmetrical biconcave & $48(52)$ & $0.19(0.20-0.53)$ \\
\hline & Dorsal thicker than volar & $41(45)$ & \\
\hline & Volar thicker than dorsal & $3(3)$ & \\
\hline \multicolumn{4}{|c|}{ Dorsal radioulnar ligament } \\
\hline \multirow[t]{3}{*}{ Sagittal morphology } & Continuous with TFC SI & $74(80)$ & $0.20(0.07-0.32)$ \\
\hline & Discontinuous with TFC SI & $9(10)$ & \\
\hline & Not able to assess & $9(10)$ & \\
\hline \multirow[t]{3}{*}{ Axial fiber continuity } & Continuous fibers & $77(84)$ & $0.06(-0.07-0.19)$ \\
\hline & Fiber discontinuation & $5(5)$ & \\
\hline & Not able to assess & $10(11)$ & \\
\hline \multicolumn{4}{|c|}{ Volar radioulnar ligament } \\
\hline \multirow[t]{3}{*}{ Sagittal morphology } & Continuous with TFC SI & $55(60)$ & $0.23(0.11-0.36)$ \\
\hline & Discontinuous with TFC SI & $23(25)$ & \\
\hline & Not able to assess & $14(15)$ & \\
\hline \multirow[t]{3}{*}{ Axial fiber continuity } & Continuous fibers & $82(89)$ & $-0.04(-0.19-0.12)$ \\
\hline & Fiber discontinuation & $1(1)$ & \\
\hline & Not able to assess & $9(10)$ & \\
\hline \multicolumn{4}{|l|}{ Proximal lamina } \\
\hline \multirow[t]{3}{*}{ Coronal homogeneity } & Homogeneous hypo-intense & $51(55)$ & $0.18(0.04-0.32)$ \\
\hline & Diffuse lamination & $33(36)$ & \\
\hline & Not able to assess & $8(9)$ & \\
\hline \multicolumn{4}{|l|}{ Distal lamina } \\
\hline \multirow[t]{3}{*}{ Coronal homogeneity } & Homogeneous hypo-intense & $44(48)$ & $0.13(-0.01-0.26)$ \\
\hline & Diffuse lamination & $39(42)$ & \\
\hline & Not able to assess & $9(10)$ & \\
\hline \multicolumn{4}{|c|}{ Ligamentum subcruentum } \\
\hline \multirow[t]{2}{*}{ Coronal morphology } & Hyper-intense signal between lamina & $74(80)$ & $0.22(0.05-0.38)$ \\
\hline & Not visible & $18(20)$ & \\
\hline \multicolumn{4}{|l|}{ Meniscus homolog } \\
\hline \multirow[t]{3}{*}{ Coronal morphology } & Diffuse hypo-intensity & $52(57)$ & $0.04(-0.08-0.16)$ \\
\hline & Clearly delineated hypo-intensity & $24(26)$ & \\
\hline & Not visible & $16(17)$ & \\
\hline \multicolumn{4}{|l|}{ Wrist position } \\
\hline \multirow[t]{3}{*}{ Axial position } & Supination & $47(51)$ & $0.62(0.53-0.84)$ \\
\hline & Neutral & $42(46)$ & \\
\hline & Pronation & $3(3)$ & \\
\hline \multicolumn{4}{|c|}{ Extensor carpi ulnaris tendon } \\
\hline \multirow[t]{3}{*}{ Axial position } & Completely within groove & $51(55)$ & $0.59(0.46-0.72)$ \\
\hline & Partially within groove & $31(34)$ & \\
\hline & Completely outside groove & $10(11)$ & \\
\hline
\end{tabular}


Table 2 (continued)

\begin{tabular}{|c|c|c|c|}
\hline Scoring item & MRI characteristics & Frequency $(\%)$ & Kappa $(95 \%$ CI) \\
\hline \multirow[t]{2}{*}{ Axial peri-tendinous SI } & Hypo-intense/intermediate & $48(52)$ & $0.22(0.05-0.38)$ \\
\hline & Focal increased & $44(48)$ & \\
\hline \multirow[t]{2}{*}{ Axial intra-tendinous SI } & Homogeneous hypo-intense & $49(53)$ & $0.29(0.12-0.45)$ \\
\hline & Focal or linear increased & $43(47)$ & \\
\hline \multicolumn{4}{|l|}{ Joint effusion } \\
\hline \multirow[t]{4}{*}{ Prestyloid recess } & Conical shaped & $37(40)$ & $0.31(0.21-0.42)$ \\
\hline & Tubular shaped & $17(18)$ & \\
\hline & Saccular shaped & $10(11)$ & \\
\hline & Not visible & $28(30)$ & \\
\hline \multirow[t]{3}{*}{ DRUJ effusion } & Absent & $22(24)$ & $0.30(0.18-0.43)$ \\
\hline & Small amount & $57(61)$ & \\
\hline & Substantial amount & $12(14)$ & \\
\hline \multirow[t]{3}{*}{ PTJ effusion } & Absent & $22(24)$ & $0.43(0.31-0.55)$ \\
\hline & Small amount & $44(48)$ & \\
\hline & Substantial amount & $26(28)$ & \\
\hline \multirow[t]{3}{*}{ Ulnar-sided cysts } & Absent & $81(88)$ & $0.36(0.23-0.50)$ \\
\hline & Present volar & $8(9)$ & \\
\hline & Present dorsal & $3(3)$ & \\
\hline
\end{tabular}

$D R U J$ distal radioulnar joint, $P T J$ pisotriquetral joint, $T F C$ triangular fibrocartilage, $S I$ signal intensity

types of homogeneity that were scored included slight morphological variations on this vertical line of increased signal intensity. Inter-observer agreement for coronal homogeneity was poor $(\mathrm{ICC}=0.13,95 \% \mathrm{CI}=0.16-0.24)$.

\section{Dorsal and volar radioulnar ligaments (RUL)}

In the sagittal plane, the dorsal and volar RUL were scored as continuous and indiscernible structures with the TFC in 74 $(80 \%)$ and $55(60 \%)$ observations, respectively, while the volar RUL signal intensity appeared to be discontinuous with the
TFC in $23(25 \%)$ observations (Fig. 3). The inter-observer agreement was fair $(\mathrm{ICC}=0.23,95 \% \mathrm{CI}=0.11-0.36)$ for volar RUL and poor ( $\mathrm{ICC}=0.20,95 \% \mathrm{CI}=0.07-0.32$ ) for dorsal RUL sagittal morphology. Axial RUL fiber continuity was scored as continuous in $77(84 \%)$ observations of the dorsal RUL and $82(89 \%)$ of the volar RUL. In 5 (5\%) observations, the dorsal RUL showed fiber discontinuation, for the volar RUL fiber discontinuation was seen in 1 (1\%) observation. For this item, inter-observer agreement regarding the dorsal and volar RUL was poor $(\mathrm{ICC}=0.06,95 \% \mathrm{CI}=-0.07-0.19$ and $\mathrm{ICC}=-0.04,95 \% \mathrm{CI}=-0.19-0.12$, respectively).
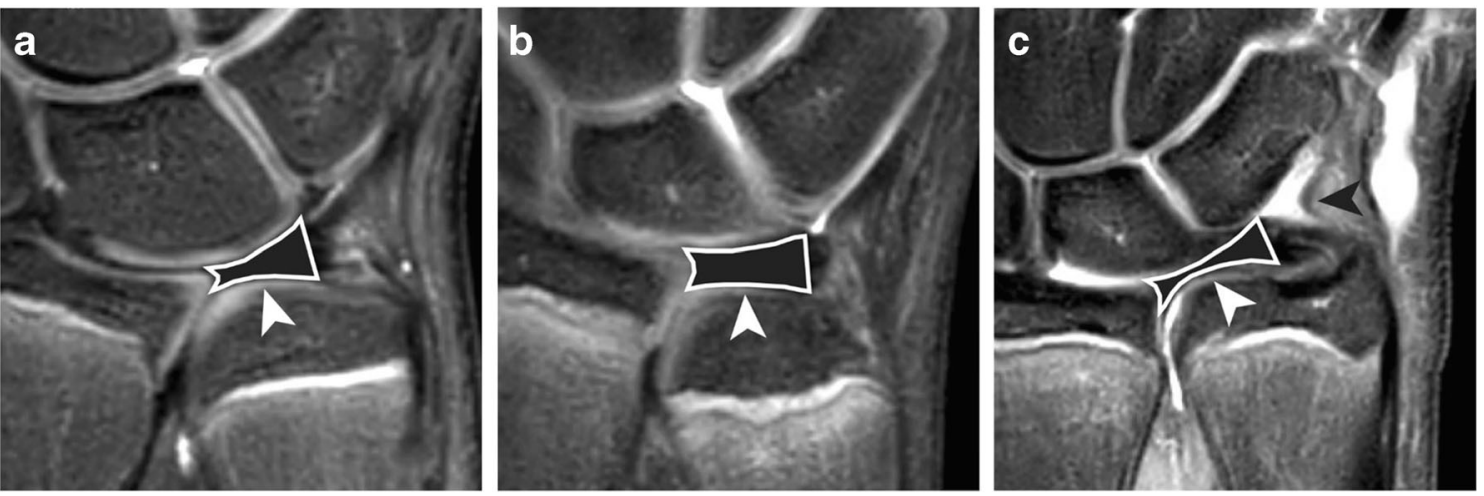

Fig. 1 Coronal proton-density weighted fat-saturated MRI showing TFC morphology delineated in white and indicated by the white arrowheads a as a slightly radially tilted asymmetrical bowtie, $\mathbf{b}$ as a shorter, thicker and more horizontal structure, and $\mathbf{c}$ as a thinner and more stretched structure.
Image $\mathbf{c}$ also shows the meniscus homolog morphology as a diffuse triangular shaped hypo-intensity (black arrowhead) directly lateral from the prestyloid recess 
Fig. 2 Coronal proton-density weighted fat-saturated MRI showing TFCs (indicated with arrowheads) with a hypo-intense signal intensity, b diffuse hyperintense signal intensity not extending through the articular surface, c vertical linear hyperintense signal intensity with discontinuation of the disc, and $\mathbf{d}$ a broader variation on this vertical line. Image a also shows the proximal (short arrow) and distal lamina (long arrow) as homogeneous hypo-intense bands, separated by the ligamentum subcruentum shown as a hyperintense signal intensity (asterisk)
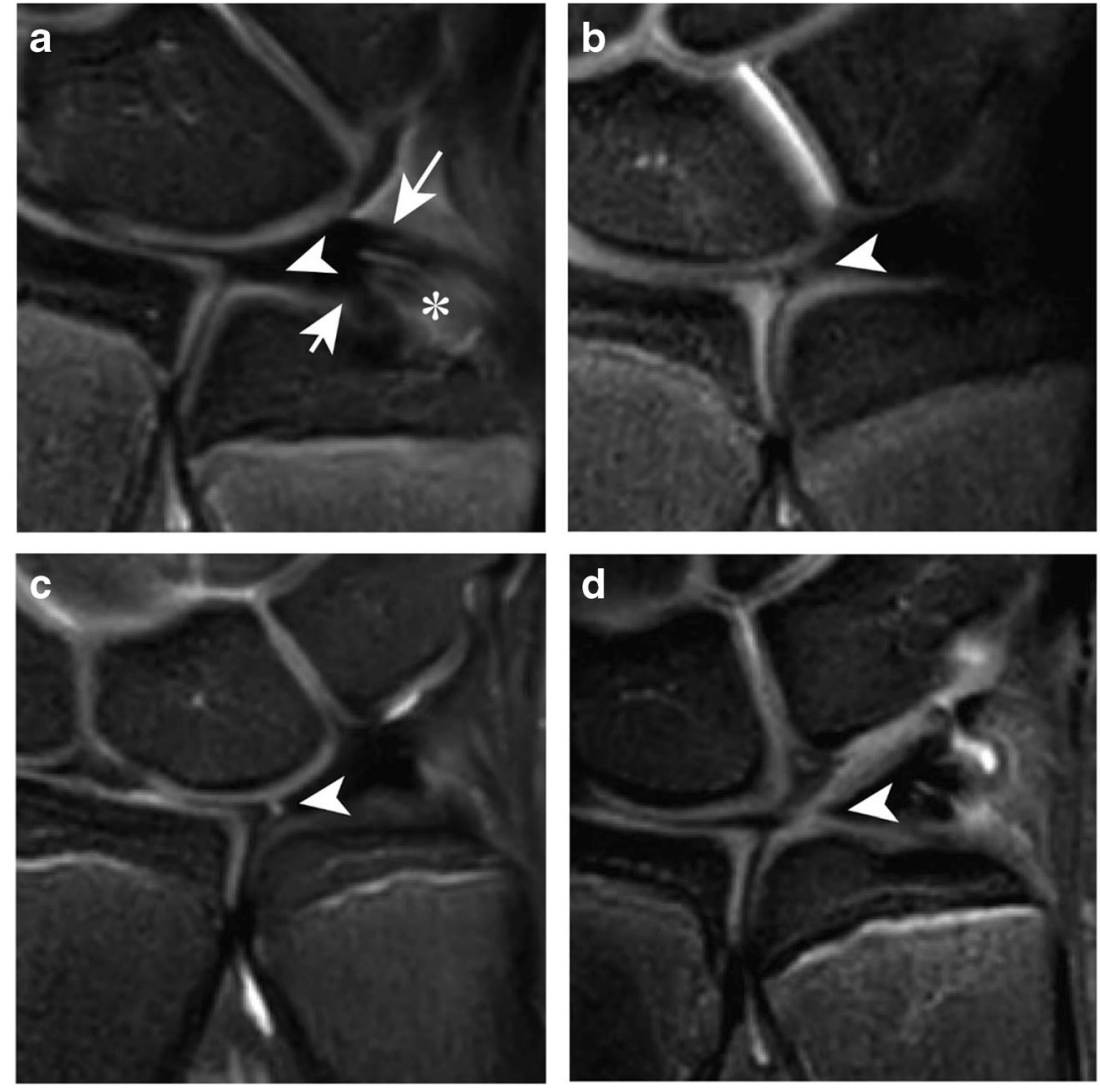

\section{Proximal (deep) and distal (superficial) lamina}

Homogeneity of the proximal and distal lamina on the coronal view was scored as homogeneous hypo-intense in $51(55 \%)$ (Fig. 2a) and 44 (48\%) observations, respectively. In 33 (36\%) of the proximal and $39(42 \%)$ of the distal lamina, a diffuse lamination with hyper-intense signals was seen. Inter-rater agreement for both proximal and distal lamina coronal homogeneity was poor $(\mathrm{ICC}=0.18,95 \% \mathrm{CI}=0.04-0.32$ and $\mathrm{ICC}=0.13,95 \% \mathrm{CI}=-0.01-0.26$, respectively).

\section{Ligamentum subcruentum}

Coronal morphology of the ligamentum subcruentum was identified as a hyper-intense signal intensity in between the proximal and distal lamina in $74(80 \%)$ observations (see Fig. 2a). In 18 (20\%) observations, this structure was not clearly visible. Agreement for this item was fair $(\mathrm{ICC}=$ $0.22,95 \% \mathrm{CI}=0.05-0.38)$.

\section{Meniscus homolog (MH)}

Meniscus homolog morphology on the coronal view was scored as a diffuse triangular shaped hypo-intensity directly ulnar from the prestyloid recess in $52(57 \%)$ observations (Fig. 1c). In $24(26 \%)$ observations, this triangular-shaped hypo- intensity was clearly demarcated. The meniscus homolog was not discernable and therefore scored as not visible in 16 (17\%) observations. Inter-observer agreement was poor $(\mathrm{ICC}=0.04,95 \% \mathrm{CI}=-0.08-0.16)$.

\section{TFCC-related features}

The results of additional TFCC-related MRI characteristics of the ulnar side of the wrist are also shown in Table 2.

\section{Wrist and ECU tendon position}

Neutral wrist position based on the axial plane was seen in 42 (46\%) observations. The wrist was defined as supinated in 47 (51\%) observations and pronated in $3(3 \%)$. The ECU tendon position was scored as completely positioned within its groove in $51(55 \%)$, as partially within its groove in 31 (34\%), and as completely dislocated out of its groove in 10 (11\%) observations (Fig. 4). The distribution of ECU tendon positions significantly differed between a supinated and a nonsupinated wrist position ( $p=0.031$, Table 3 ). Inter-rater agreement for wrist and ECU tendon position was, respectively, substantial ( $\mathrm{ICC}=0.62,95 \% \mathrm{CI}=0.53-0.84)$ and moderate $(\mathrm{ICC}=0.59,95 \% \mathrm{CI}=0.46-0.72)$. 
Fig. 3 Sagittal proton-density weighted MRI showing the volar RUL as a structure that is a continuous and indiscernible with the TFC (arrowhead) and b discontinuous with the TFC (arrowhead) in between the ulnar head (diamonds) and lunate bone (asterisks)
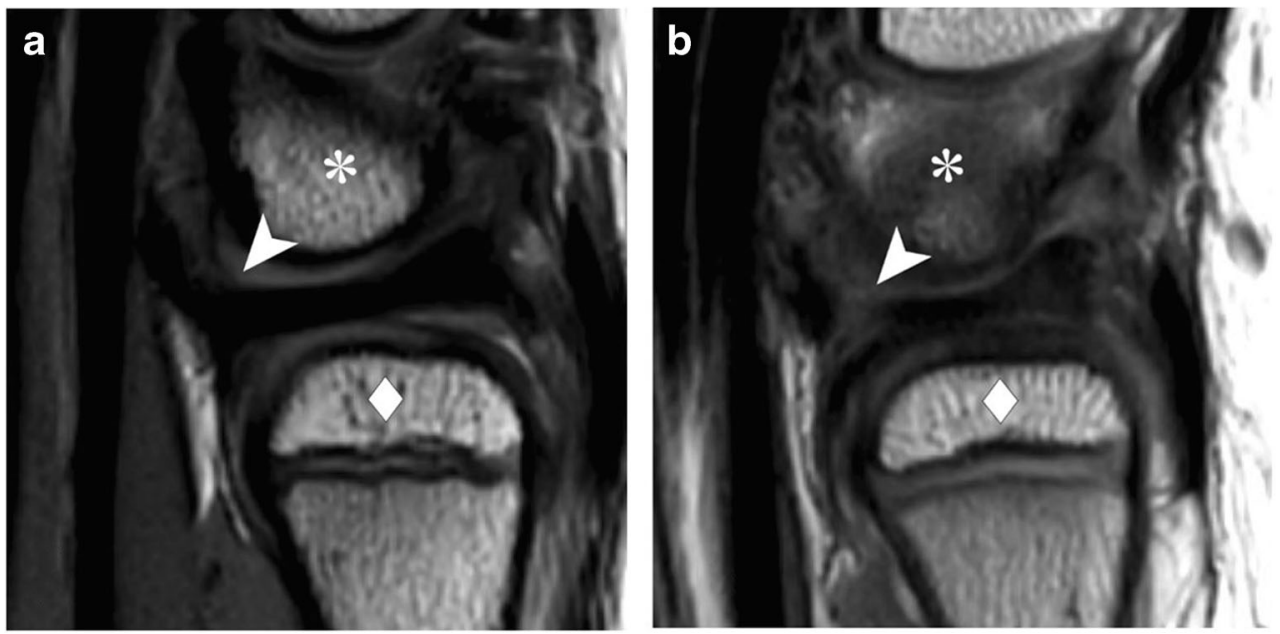

\section{ECU tendon signal intensity}

The signal intensity surrounding and inside the ECU tendon in the axial plane was assessed on the slices in between the proximal base of the ECU groove and the distal border of the extensor retinaculum. The signal intensity was scored as hypo-intense to intermediate in $48(52 \%)$ and $49(53 \%)$ observations, respectively. In $44(48 \%)$ of the peri-tendinous and $43(47 \%)$ of the intra-tendinous signal intensities, focal or linear increased signal intensity was observed. Peritendinous increased signal was located proximal from the styloid process in $34(77 \%)$ observations. Intra-tendinous increased signal was located at the styloid level in $29(67 \%)$. Inter-observer agreement for scoring ECU tendon signal intensity was fair for peri-tendinous $(\mathrm{ICC}=0.22,95 \% \mathrm{CI}=$ $0.05-0.38)$ as well as intra-tendinous ( $\mathrm{ICC}=0.29,95 \% \mathrm{CI}=$ $0.12-0.45$ ) signal intensity.

\section{Joint effusion}

Coronal morphology of the prestyloid recess was scored as a conical shape in 37 (40\%) observations, a tubular shape in 17 (18\%), and a saccular shape in 10 (11\%) (Fig. 5). In $23(30 \%)$ assessments, the prestyloid recess itself was not visible. A fair ( $\mathrm{ICC}=0.31,95 \% \mathrm{CI}=0.21-0.42$ ) inter-observer agreement for this item was found. A small amount of distal radioulnar joint (DRUJ) and pisotriquetral joint (PTJ) effusion was present according to $37(40 \%)$ and $44(48 \%)$ observations, respectively. In $12(14 \%)$ and $26(28 \%)$ observations, a substantial amount of DRUJ and PTJ effusion was identified, respectively. Inter-observer agreement was fair $(\mathrm{ICC}=0.30,95 \% \mathrm{CI}=$ $0.18-0.43$ ) for DRUJ effusion and moderate ( $\mathrm{ICC}=0.43$, $95 \% \mathrm{CI}=0.31-0.55)$ for PTJ effusion. Additional ulnarsided cysts were identified at the volar side in $8(9 \%)$ and on the dorsal side in $3(3 \%)$ observations, with a fair $(\mathrm{ICC}=0.36$, $95 \% \mathrm{CI}=0.23-0.50$ ) inter-observer agreement.

\section{Other relevant findings}

Two observers noticed physeal edema in the distal radius and ulna from one participant and three observers noticed a type two lunate bone with bone marrow edema in one participant. Besides several radial and carpal ganglion cysts, no other relevant findings were observed.

\section{Discussion}

Knowledge regarding TFCC characteristics on MRI in adolescents is lacking in the literature. In this study, a wide range in variation of individual TFCC components and TFCC-related features on MRI was observed in healthy
Table 3 Contingency table for the position of the extensor carpi ulnaris tendon regarding the ulnar groove and wrist position during MRI expressed in frequencies with percentages

\begin{tabular}{lllll}
\hline & \multicolumn{4}{l}{ Position extensor carpi ulnaris tendon regarding ulnar groove } \\
\cline { 2 - 5 } & Completely within & Partially within & Completely outside & Total \\
\hline Supination & $22(23.9)$ & $16(17.4)$ & $9(9.7)$ & $47(51.0)$ \\
Neutral position & $26(28.3)$ & $15(16.3)$ & $1(1.1)$ & $42(45.7)$ \\
Pronation & $3(3.3)$ & $0(0.0)$ & $0(0.0)$ & $3(3.3)$ \\
Total & $51(55.4)$ & $31(33.7)$ & $10(10.9)$ & $92(100.0)$ \\
\hline
\end{tabular}



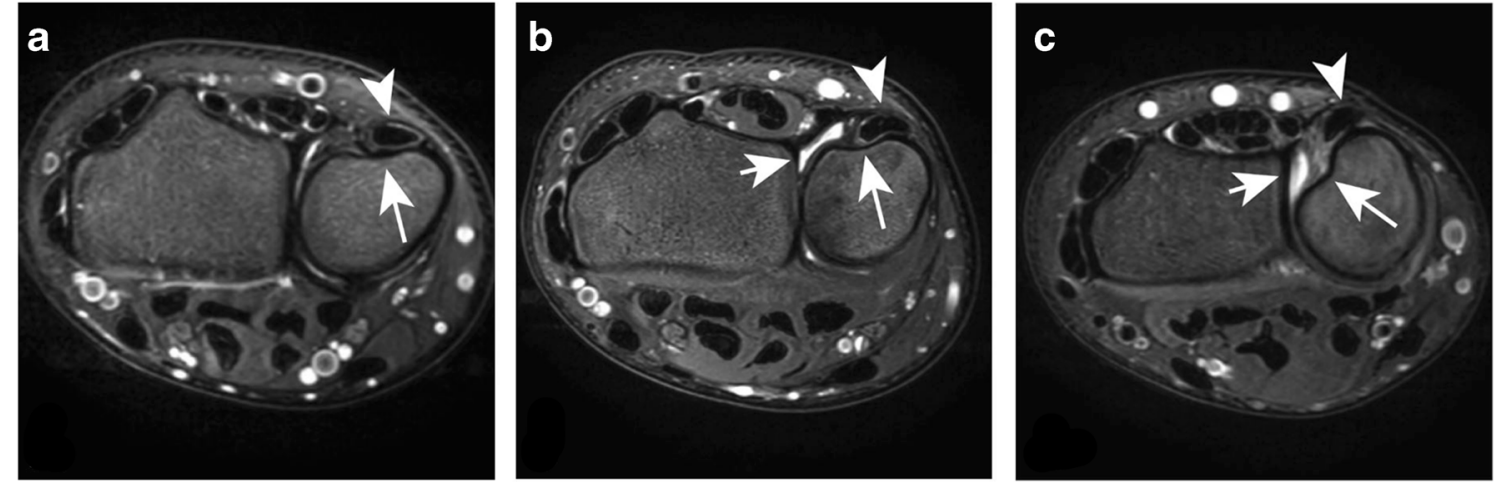

Fig. 4 Axial T2 weighted fat-saturated MRI showing the ECU tendon (arrowheads) position a completely within, b partially within, and c completely outside its groove (long arrows). All three wrist positions

asymptomatic adolescents. Generally, the TFC appeared as a slightly radially tilted bowtie with homogeneous hypointense signal intensity in coronal view and as a symmetrical biconcave disc in the sagittal view. The RULs were mostly continuous with TFC. The proximal and distal lamina was homogeneously hypo-intense or diffusely laminated and separated by a hyper-intense ligamentum subcruentum. The meniscus homolog was a diffuse triangular shaped hypo-intensity lateral from the prestyloid recess.

The coronal morphology of the disc was observed as thinner and more stretched in $13 \%(\mathrm{ICC}=0.33,95 \% \mathrm{CI}=0.20$ 0.46 ) of the observations. Literature has suggested that this stretched shape of the disc is associated with a positive ulnar variance [27]. Yet, the present study did not find a statistical difference in ulnar variance when the stretched morphology was scored. Probably, this can be explained by the small numbers of observations of this stretched shape $(n=12)$. Neither did we find a statistical difference in wrist position, which is in accordance with a previous observation that shape of the disc did not change during pro- or supination [28].

A diffuse increased signal intensity within the disc, considered a sign of TFC degeneration in adults (Palmer 2A injury), was seen in $33 \%(\mathrm{ICC}=0.13,95 \% \mathrm{CI}=0.16-0.24)$ of the were scored as a supinated wrist position with the ulnar head in dorsal rotation. Image $\mathbf{b}$ and $\mathbf{c}$ also show a substantial amount of DRUJ effusion (short arrows)

observations [29]. Since the young participants did not perform wrist-loading sports more than twice a week, this highly unlikely represents degeneration. Also, a vertical linear increased signal intensity of the TFC with discontinuation of the disc, currently interpreted as a traumatic TFC perforation (Palmer 1A injury), was seen in 20\% of observations [24].

It is known that newborns have higher percentages of vascularized peripheral TFC margins than adults [30]. On MRI of the knee in children, similar signal intensities within the menisci that tended to decrease with age were found and authors suggest they might reflect normal increased vascularization [31]. Since TFC vascularization appears to resemble menisci, this could be an explanation for both observed TFC signal intensity changes. However, it is difficult to distinguish the vertical hyper-intense lines that appear as perforations from vascularity in this study.

An early study on TFC perforations that is still frequently referred to did not observe perforations in the first two decades of life [32]. Even though a subsequent study on gross anatomy of fetal and newborn cadaveric wrists reported TFC perforations in 27 out of $120(22.5 \%)$ wrists, this type of "injury" is still used as indication for arthroscopic debridement in pediatric patients with persistent wrist pain [33, 34]. We found a comparable incidence of signal intensities imposing as TFC
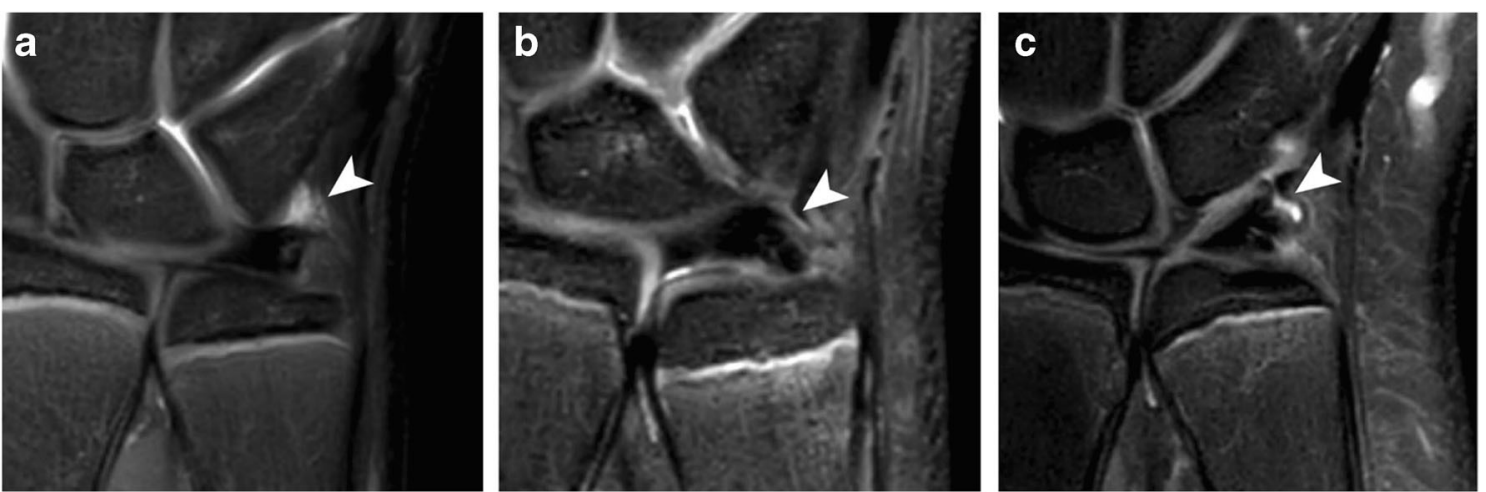

Fig. 5 Coronal proton-density weighted fat-saturated MRI showing the a conical shaped prestyloid recess (arrowhead), b tubular shaped prestyloid recess (arrowhead), and c saccular shaped prestyloid recess (arrowhead) 
perforations, yet future studies should demonstrate the anatomical substrate of these linear signal intensity changes.

With the recent development of MRI techniques, additional TFCC abnormalities can be visualized [24]. Discontinuation between the dorsal and volar edges of the TFC with the joint capsule is currently suggested as a pathological capsular detachment and as an additional type of Palmer injury [24]. In the present study, a discontinuation of the volar RUL with the TFC due to a linear hyper-intense signal intensity was found in $23(25 \%)$ observations mimicking this capsular detachment. In the axial plane, however, only one observer found fiber discontinuation of the volar radioulnar ligament. Therefore, we can conclude that this observation rather reflected a volume averaging artifact than a true fiber discontinuation. This stresses the importance of using both imaging planes when assessing the radioulnar ligaments.

We observed a high prevalence of ECU tendon (sub)luxation (44\%), which is even higher than reported by a recent study in 123 asymptomatic adults that observed $30 \%$ of ECU tendon (sub)luxation [35]. Mild ECU tendon subluxation is frequently observed in the supinated wrist; however, rupture of the ECU subsheath is considered to cause greater subluxation in supination [36]. In the present study, $51 \%$ of the wrists were scanned in a supinated position. Additionally, $90 \%$ of the wrists with complete ECU tendon luxation were identified as supinated. Therefore, in case of a subluxation or complete luxation of the ECU tendon in adolescents, this should not be associated with ECU tendon subsheath injury if scanned in a supinated position.

The high rate of supination in the present study is a limitation, especially since a neutral wrist position is key in assessing the dorsal and volar RUL [5]. In line with current clinical practice, the general adult wrist coils were used. Despite careful neutral positioning prior to scanning, this probably enabled increased moving space after placement in neutral position. We therefore recommend additional elbow support and focus on fixation in MRI wrists involving adolescents. Another limitation was the fact that observers were aware of the asymptomatic character of the participants. However, the effect of possible interpretation bias was limited since we intended to report imaging observations rather than interpretations on pathology.

Despite multiple consensus meetings and a calibration session, the inter-observer agreement in TFCC assessment was rather low. However, due to our diligence in designing the scoring items, we do not consider this to reflect a limited methodology, but rather the personal interpretation that TFCC assessment in clinical practice is largely subject to $[10,13]$. We believe that variation in structural terminology of TFCC components across studies is a major complicating factor and agree that clinical practice would benefit from consensus on TFCC definitions and abnormality criteria [8]. Especially, since wrist MRIs change the suspected clinical diagnosis in $46 \%$ of predominantly surgical referrals in pediatric patients under 18 years and even lead to a change in therapeutic strategy in $86 \%$ [37]. This, in combination with a high rate of asymptomatic MRI findings of the TFCC, bears the risk of unnecessary wrist arthroscopy and emphasizes the importance of multidisciplinary decision-making.

In conclusion, the present study showed that asymptomatic MRI findings, whether normal variation or asymptomatic abnormalities, can be observed in individual TFCC components and TFCC-related features of healthy asymptomatic adolescents. MRI findings that can be interpreted as abnormalities such as TFC perforations, TFC degeneration, pathological capsular detachment of the volar RUL, and ECU tendon (sub)luxation indicating ECU subsheath injury were observed. It is important to consider these asymptomatic findings when interpreting MRIs of the wrist in symptomatic pediatric patients and deciding upon arthroscopic treatment.

Supplementary Information The online version contains supplementary material available at https://doi.org/10.1007/s00256-021-03779-8.

\section{Acknowledgments None.}

\section{Declarations}

Conflict of interest The authors declare that they have no conflict of interest.

Ethical approval All procedures performed in studies involving human participants were in accordance with the ethical standards of the institutional and/or national research committee and with the 1964 Helsinki declaration and its later amendments or comparable ethical standards.

Informed consent Informed consent was obtained from all individual participants included in the study.

\section{References}

1. Palmer AK. Triangular fibrocartilage complex lesions: a classification. J Hand Surg Am. 1989;14(4):594-606.

2. Terry CL, Waters PM. Triangular fibrocartilage injuries in pediatric and adolescent patients. J Hand Surg Am. 1998;23(4):626-34.

3. Yamabe E, Nakamura T, Pham P, Yoshioka H. The athlete's wrist: ulnar-sided pain. Semin Musculoskelet Radiol. 2012;16(4):331-7.

4. Watanabe A, Souza F, Vezeridis PS, Blazar P, Yoshioka H. Ulnarsided wrist pain. II. Clinical imaging and treatment. Skelet Radiol. 2010;39(9):837-57.

5. Jens S, Luijkx T, Smithuis FF, Maas M. Diagnostic modalities for distal radioulnar joint. J Hand Surg Eur Vol. 2017;42(4):395-404.

6. Sachar K. Ulnar-sided wrist pain: evaluation and treatment of triangular fibrocartilage complex tears, ulnocarpal impaction syndrome, and lunotriquetral ligament tears. J Hand Surg Am. 2012;37(7):1489-500.

7. Lee JK, Hwang JY, Lee SY, Kwon BC. What is the natural history of the triangular fibrocartilage complex tear without distal radioulnar joint instability? Clin Orthop Relat Res. 2018. 
8. Chan JJ, Teunis T, Ring D. Prevalence of triangular fibrocartilage complex abnormalities regardless of symptoms rise with age: systematic review and pooled analysis. Clin Orthop Relat Res. 2014;472(12):3987-94.

9. Bendre HH, Oflazoglu K, van Leeuwen WF, Rakhorst H, Ring D, Chen NC. The prevalence of triangular fibrocartilage complex signal abnormalities on magnetic resonance imaging relative to clinical suspicion of pathology. J Hand Surg Am. 2018;43(9):819-26 e811.

10. Treiser MD, Crawford K, Iorio ML. TFCC injuries: meta-analysis and comparison of diagnostic imaging modalities. J Wrist Surg. 2018;7(3):267-72.

11. Blazar PE, Chan PS, Kneeland JB, Leatherwood D, Bozentka DJ, Kowalchick R. The effect of observer experience on magnetic resonance imaging interpretation and localization of triangular fibrocartilage complex lesions. J Hand Surg Am. 2001;26(4):742-8.

12. Anderson ML, Skinner JA, Felmlee JP, Berger RA, Amrami KK. Diagnostic comparison of 1.5 Tesla and 3.0 Tesla preoperative MRI of the wrist in patients with ulnar-sided wrist pain. J Hand Surg Am. 2008;33(7):1153-9.

13. Iordache SD, Rowan R, Garvin GJ, Osman S, Grewal R, Faber KJ. Prevalence of triangular fibrocartilage complex abnormalities on MRI scans of asymptomatic wrists. J Hand Surg Am. 2012;37(1): 98-103.

14. Davis KW, Gill KG. Upper extremity injuries in young athletes. Semin Musculoskelet Radiol. 2012;16(4):269-79.

15. Farr S, Grill F, Ganger R, Graf A, Girsch W. Pathomorphologic findings of wrist arthroscopy in children and adolescents with chronic wrist pain. Arthroscopy. 2012;28(11):1634-43.

16. Ramavath AL, Unnikrishnan PN, George HL, Sathyamoorthy P, Bruce CE. Wrist arthroscopy in children and adolescent with chronic wrist pain: arthroscopic findings compared with MRI. J Pediatr Orthop. 2017;37(5):e321-5.

17. Pfanner S, Diaz L, Ghargozloo D, Denaro V, Ceruso M. TFCC lesions in children and adolescents: open treatment. J Hand Surg Asian Pac. 2018;23(4):506-14.

18. Fishman FG, Barber J, Lourie GM, Peljovich AE. Outcomes of operative treatment of triangular fibrocartilage tears in pediatric and adolescent athletes. J Pediatr Orthop. 2018;38(10):e618-22.

19. Farr S, Schuller M, Ganger R, Girsch W. Outcomes after arthroscopic debridement of the triangular fibrocartilage complex in adolescents. J Wrist Surg. 2018;7(1):43-50.

20. Farr S, Zechmann U, Ganger R, Girsch W. Clinical experience with arthroscopically-assisted repair of peripheral triangular fibrocartilage complex tears in adolescents-technique and results. Int Orthop. 2015;39(8):1571-7.

21. Trehan SK, Schimizzi G, Shen TS, Wall LB, Goldfarb CA. Arthroscopic treatment of triangular fibrocartilage complex injuries in paediatric and adolescent patients. J Hand Surg Eur Vol. 2019;44(6):582-6.

22. Kox LS, Kraan RBJ, van Dijke KF, Hemke R, Jens S, de Jonge $\mathrm{MC}$, et al. Systematic assessment of the growth plates of the wrist in young gymnasts: development and validation of the Amsterdam MRI assessment of the Physis (AMPHYS) protocol. BMJ Open Sport Exerc Med. 2018;4(1):e000352.
23. Kox LS, Jens S, Lauf K, Smithuis FF, van Rijn RR, Maas M. Wellfounded practice or personal preference: a comparison of established techniques for measuring ulnar variance in healthy children and adolescents. Eur Radiol. 2019.

24. Zhan H, Zhang H, Bai R, Qian Z, Liu Y, Zhang H, et al. Highresolution 3-T MRI of the triangular fibrocartilage complex in the wrist: injury pattern and MR features. Skelet Radiol. 2017;46(12): $1695-706$

25. von Borstel D, Wang M, Small K, Nozaki T, Yoshioka H. Highresolution 3T MR imaging of the triangular fibrocartilage complex. Magn Reson Med Sci. 2017;16(1):3-15.

26. Landis JR, Koch GG. The measurement of observer agreement for categorical data. Biometrics. 1977;33(1):159-74.

27. Vezeridis PS, Yoshioka H, Han R, Blazar P. Ulnar-sided wrist pain. Part I: anatomy and physical examination. Skelet Radiol. 2010;39(8):733-45.

28. Pfirrmann CW, Theumann NH, Chung CB, Botte MJ, Trudell DJ, Resnick D. What happens to the triangular fibrocartilage complex during pronation and supination of the forearm? Analysis of its morphology and diagnostic assessment with MR arthrography. Skelet Radiol. 2001;30(12):677-85.

29. Burns JE, Tanaka T, Ueno T, Nakamura T, Yoshioka H. Pitfalls that may mimic injuries of the triangular fibrocartilage and proximal intrinsic wrist ligaments at MR imaging. Radiographics. 2011;31(1):63-78.

30. Mikic Z. The blood supply of the human distal radioulnar joint and the microvasculature of its articular disk. Clin Orthop Relat Res. 1992;275:19-28.

31. Takeda Y, Ikata T, Yoshida S, Takai H, Kashiwaguchi S. MRI high-signal intensity in the menisci of asymptomatic children. J Bone Joint Surg Br. 1998;80(3):463-7.

32. Mikic ZD. Age changes in the triangular fibrocartilage of the wrist joint. J Anat. 1978;126(Pt 2):367-84.

33. Wu M, Miller PE, Waters PM, Bae DS. Early results of surgical treatment of triangular fibrocartilage complex tears in children and adolescents. J Hand Surg Am. 2019.

34. Tan AB, Tan SK, Yung SW, Wong MK, Kalinga M. Congenital perforations of the triangular fibrocartilage of the wrist. J Hand Surg Br. 1995;20(3):342-5.

35. Luijkx T, Buckens CF, van Seeters T, Pegge SA, Maas M. ECU tendon subluxation: a nonspecific MRI finding occurring in all wrist positions irrespective of ulnar-sided symptoms? Eur J Radiol. 2019;116:192-7.

36. Ghatan AC, Puri SG, Morse KW, Hearns KA, von Althann C, Carlson MG. Relative contribution of the subsheath to extensor carpi ulnaris tendon stability: implications for surgical reconstruction and rehabilitation. J Hand Surg Am. 2016;41(2):225-32.

37. Taylor KW, Moore MM, Brian J, Methratta S, Bernard S. Wrist MR imaging in children: effect on clinical diagnosis and management. Clin Imaging. 2017;44:61-5.

Publisher's note Springer Nature remains neutral with regard to jurisdictional claims in published maps and institutional affiliations. 